l'indicateur je veux dire si l'on masque la zone pour laquelle la décoloration du jaune est lentement progressive, on obtient un virage net. J'ai employé pour cela le bleu de méthylène et l'on a un virage du vert (alcalin) au bleu ciel (acide).

Pour avoir l'acide lastique occlus dans la masse on doit dissoudre la caséine, comme pour un dosage de matière grasse. Or on ne peut ici le faire avec un aside. Il faudrait un aside su'fisamment fort et, qui en tou cas, dézomposerait le lactique. Il faut donc avoir recours à la soude. Comme pour dégrader (ou dissoudre) cette caséine, il faudra être en zone al zaline, nous reviendrons en arrière par un titrage en retour avec un acide.

Et je propose une méthode ou plutôt une modification à des méthodes déjà existantes :

10 grammes de caséine finement moulue sont portés avec $100 \mathrm{~cm}^{3}$ d'eau distillée à l'ébullition.

On additionne peu à peu 20 ou $25 \mathrm{~cm}^{3}$ exactement mesurés de soude Dornic jusqu'à ce que la masse forme un liquide visqueụx (à chaud) ne présentant plus de grain en suspension.

On fait tomber $\mathrm{X}$ gouttes de solution de paranitrophénol dans l'éther a 20 grammes par litre et 5 gouttes de solution de bleu de méthyl ne à 0,5 par litre. L'ensemble est vert.

On ajoute jusqu'à virage au bleu un acide dont on connaitra la correspondance avec la soude Dornic (si l'on prend l'acide chlorydrique on le règlera à 4,05 pour un litre, de façon qu'il y ait correspondance exacte : $1 \mathrm{~cm}^{3}$ de l'un pour $1 \mathrm{~cm}^{3}$ de l'autre).

Soit $\mathrm{N}$ le nombre de $\mathrm{cm}^{3}$ de soude Dornic ajoutée, $\mathrm{n}$ le nombre de $\mathrm{cm}^{3}$ d'acide.

$$
\frac{(\mathrm{N}-\mathrm{n})}{10}=\mathrm{x} \mathrm{g} \text {. d'acide lactique } \% \text {. }
$$

\title{
L'INFLUENCE DU LAB-FERIMENT SUR LA MATURATION DU FROMAGE. EXPÉRIENCES AVEC LE BACKSTEIN RUSSE.
}

\author{
par J. HAWESSON, Agronome en Laiterie \\ (Travail de l'Institut de Laiterie de Wologda, Russie du Nord)
}

(Suite)

Ainsi, dans le travail ci-devant cité, ORLA-JENSEN admettait l'action de la pepsine même en milieu alsalin au tournesol. Plus tard (1906), se fondant sur les expériences faites par lui et mal réussies -ur l'accélération de la maturation du fromage $\mathrm{d}^{\mathbf{E}} \mathrm{Emmenthal,} \mathrm{au} \mathrm{moyen} 1$ addition de la pepsine, ainsi que sur les expériences déjà mentionnées de Spar LAN. zani et Bertozzi avec le Parmesan (1905), ORLA-JENSEn a brusquen'ent changé son opinion en refusant d'attribuer un rôle à. la pepsine, du moins dans la fabrication du fromage d'Emmenthal, en expliquant cela par 
l'affaiblissement du ferment lors du second réchauffement qui se fait à température élevée et qui est de longue durée (1).

En 1907, Mue van Herwerden [12] a montré que par une action prolongée durant 14 jours entiers, le lab commercial est susceptible d'agir protéolytiquement sur le blane d'œuf de poule en milieu neutre au tournesol.

Un peu avant, Perry (2) avait constaté que l'action du lab ne s'en tient à la formation de la paracaséine. Il a attribué cette action à un nouveau ferment protéolytique " sévèrement spécifique pour la caséine ».

Vers ce moment (1907), ORLA-JENSEN se mit à modifier définitivement ses conclusions de 1904, en proclamant que la pepsine est peu activée même au de gré d'acidité qu'on observe dans les fromages mous acides et que par conséquent lors de la maturation des fromages à pâte ferme beaucoup moins acides, elle ne doit pas intervenir. Voir LöHNIs [15].

Pourtant, le fait de la peptonisation croissante de la masse fromagère sous l'influence du lab ajouté avait été observé plusieurs fois comme nous l'avons vu, par toute une rangée d'expérimentateurs. Et ORLAJENSEN a tranché à sa guise le noud gordien ; il a proc lamé que l'enzyme de Petry, la " caséase " est le producteur principal des matières azotées solubles du fromage ; ajoutons qu'on ne s'est pas préoccupé de savoir si l'enzyme nouvelle accompagne toujours le lab, et si elle résiste mieux que la pepsine à la chaleur (3).

Avant de passer à l'examen des opinions sur le rôle du labferment dans le processus de la maturation des fromages, portons notre attention sur le travail bien intéressant des savants belges, L. Marcas et C. Huyge [11], publié en 1906 sous le titre : "Influence de la pepsine sur la maturation du fromage de Herve \%. Le fromage de Herve est nommé backstein français par LöHNIs [15]. Les auteurs belges ont employé les noms "fromage de Herve" et "fromage de Limbourg ", comme synonymes. Parmi les espèces des fromages, dont la maturation a été étudiée en relation avec les différentes quantités de ferments protéolytiques qu'ils peuvent contenir, le fromage de Limbourg à pâte plus ferme et le fromage de Herve, sont voisins du backstein russe auquel est consacrée la partie expérimentale de ce travail.

Le rapport de l'azote soluble à l'azote tota dans le fromage de Limbourg de cuisson d'automne; âgé de 53 jours et tenu pour mûr, est selon Marcas et HuYGE, $24,48 \%$ dans la partie extérieure du fromage et $24,68 \%$ à l'intérieur. Le backstein russe, selon mes recherches, contient à l'âge de 60 jours, $22,2 \%$ d'azote soluble par rapport à l'azote total. Selon les données de G. S. Inichoff [29], ("Annales de 1'Institut

(1) Cité d'après van Dam [21] (1915).

(2) Cité d'après van DaM [14] (1910).

(3) Cité d'après van DAM $[21]$ (1915). 
de Laiterie à Wologda ". Vol. II, N. 4. p. 103) à l'âge de 50 jours, il contient $18,8 \%$; et à l'état mûr, âgé de 85 jours : $25,3 \%$. Son degré de maturité est done 0.253. Le fromage de Herve de cuisson du printemps se comporte autrement. Son degré de maturité pour la couche extérieure, à l'âge de 49 jours est 0,548 et pour la partie interne : 0,363 . Les fromages de cette sorte ne peuvent pas être assimilés par ce caractère au backstein russe.

L3s expériences de MARCas et HoYGE on' porté sur deux séries dans chacune desquelles on a examiné 16 fromages. Ceux de la première série ont été fabriqués fin avril, ceux de la seconde, fin octobre. Les caractères de la maturation des fromages de la première et de la seconde séries ont été bien différents ; les fromages de la cuisson d'automne ont manifesté une acidité plus faible et une solubilisation des matières azotées plus lente.

Le mode opératoire a été le suivant: On partageait le lait, destiné à la euisson des fromages mis en expérience, en 4 lots égaux. Dans un premier lot, on coagulait par la voie ordinaire; mais aux trois lots restants on ajoutait, outre la quantité normale de lab employé des quantités croissantes de papsine, notamment $0,5,1,0$ et 1,5 partie pour 1.000 parties de caillé frais dans la première série des expériences (la cuisson du printemps); ces quantités furent doublées, elles étaient respectivement de $1,0,2,0$ et 3,0 parties pour 1.000 parties de caillé frais, dans la seconde série (la euisson d'automne). La force protéolytique des préparations de pepsine avait été préalablement vérifiée par l'épreuve de la digestibilité de blane d'œuf de poule coagulé.

De temps en temps, les fromages fabriqués ont été analysés : matière sèche, acidité, azote total, azote des combinaisons solubles, azote précipitable par l'acide phosphotungstique et azote ammoniacal ont été déterminés.

Tous les dosages, excepté celui de l'aciditéavaient étéfaits d'après des méthodes qui différent peu de celles qui sont en usage aujourd'hui. La détermination de l'acidité, après trituration du fromage avec de l'eau, filtration à travers un filtre de terre poreuse, titrage par une solution alcaline normale ne donne pas de résultat sûr. C'est pourquoi nous nous abstenons de publier les données obtenues par les expérimentateurs belges concernant le changement de l'acidité du fromage sous l'influence de la pepsine. Rappclons seulement que les auteurs ont constaté que l'asidité des fromages du printemps est beaucoup plus grande que celle des fromages d'automne.

Il est intéressant de constater que, malgré les grandes quantités de pepsine ajoutées, l'accroissement plus ou moins considérable de l'azote soluble, ainsi que de l'azote des produits de décomposition précipitable par l'acide phosphotungstique, n'a été observé que dans les fromages 
du printemps; et dans les fromages d'automne, cet accroissement n'a été observé qu'à un degré bien restreint. En outre, il a été bien inégal pour les fromages de deux cuissons, surtout pour celle d'automne.

Parallèlement aux variations de l'azote soluble, l'accroissement d'azote ammoniacal a été aussi très faible.

Voici les résultats trouvés par Marcás et Huyge.

\section{TABLEAU VII}

Fromages fabriqués le 12 avril.

A. Quantités d'azote soluble pour 100 d'azote total.

\begin{tabular}{|c|c|c|c|c|c|c|c|c|}
\hline \multirow[t]{2}{*}{$\begin{array}{l}\text { Date } \\
\text { des déterminations }\end{array}$} & \multicolumn{2}{|c|}{ Fromage témoin } & \multicolumn{2}{|c|}{$\begin{array}{l}\text { Fromage avec } 0,5 \\
\text { part ie de pepsine } \\
\text { pour } 1.000 \text { parties } \\
\text { de caillé frais }\end{array}$} & \multicolumn{2}{|c|}{$\begin{array}{l}\text { Fromage avec } \\
1 \text { partie de pepsine } \\
\text { pour } 1.000 \text { parties } \\
\text { de caillé frass }\end{array}$} & \multicolumn{2}{|c|}{$\begin{array}{l}\text { Fromage a vec } 1.5 \\
\text { partie de pepsine } \\
\text { pour } 1.000 \text { part les } \\
\text { de caillé frais }\end{array}$} \\
\hline & Estérieur & Noyau & Extérieur & Noyau & Extérieur & Noyau & E stérieur & Noyau \\
\hline Lo 27 avril & 10,67 & 9,66 & 8,54 & 6,48 & 10,34 & 8,85 & 8,42 & 6,67 \\
\hline Le 12 mai.. & 15,25 & 8,95 & 24,82 & 10,79 & 25,59 & 9,03 & 29,73 & 10,89 \\
\hline Le 2 juin & 55,35 & 39,32 & 68,72 & 28,56 & 66,45 & 26,97 & 67,53 & 26,41 \\
\hline Le 15 juin & 54,83 & 36,34 & 75,87 & 49,72 & 86,52 & 75,79 & & \\
\hline
\end{tabular}

B. Quantités d'azote des produits de décomposition pour 100 d'azote total.

\begin{tabular}{ll||l|r||r|r||r|r||r|r} 
Le 27 avril ..... & 6,80 & 3,07 & 3,50 & 3,14 & 6,46 & 2,54 & 3,35 & 3,03 \\
Le 12 mai...... & 7,37 & 3,54 & 9,87 & 3,73 & 8,52 & 3,90 & 11,09 & 5,05 \\
Le 2 juin ...... & 32,59 & 22,13 & 42,90 & 15,89 & 44,81 & 15,57 & 33,06 & 20,83 \\
Le 15 juin ...... & 32,69 & 23,59 & 48,45 & 25,50 & 54,94 & 26,35 & &
\end{tabular}

C. Quantités d'azole ammoniacal pour 100 d'azote total.

\begin{tabular}{ll|r||r|r||r|r||r|r} 
Le 27 avril ..... & 1,98 & 1,84 & 1,58 & 1,81 & 1,62 & 1,51 & 1,33 & 1,56 \\
Le 12 mai...... & 1,67 & 1,16 & 2,57 & 0,81 & 1,67 & 1,00 & 1,77 & 0,69 \\
Le 2 juin...... & 2,23 & 2,01 & 2,13 & 2,03 & 1,80 & 1,79 & 1,75 & 1,77 \\
Le 15 juin..... & 8,41 & 7,14 & 7,67 & 10,00 & 7,34 & 9,03 & & \\
\hline \hline
\end{tabular}

TABLEAU VIII

Fromages fabriqués le 23 octobre.

A. Quantités d'azote soluble pour 100 d'azote total.

\begin{tabular}{|c|c|c|c|c|c|c|c|c|}
\hline \multirow[t]{2}{*}{$\begin{array}{c}\text { Date } \\
\text { des déterminations }\end{array}$} & \multicolumn{2}{|c|}{ Fromage témoin } & \multicolumn{2}{|c|}{\begin{tabular}{|} 
Fromage avec \\
partie de pepsine \\
pour 1.000 parties \\
de caillé frais
\end{tabular}} & \multicolumn{2}{|c|}{$\begin{array}{c}\text { Fromage avec } \\
2 \text { par ies de pepsine } \\
\text { pour } 1.000 \text { parties } \\
\text { de caille frais }\end{array}$} & \multicolumn{2}{|c|}{$\begin{array}{l}\text { Fromage avec } \\
\text { 3 parties de pepsine } \\
\text { pour } 1.000 \text { parties } \\
\text { de caillé frais }\end{array}$} \\
\hline & Extérieur & Noyau & Extérieur & Noyau & Extérieur & Noyau & Extérieur & Noyau \\
\hline Le $1^{\text {er }}$ & 2,38 & 1,25 & 4,18 & 4,24 & 3,59 & 1,13 & 6,09 & 3,80 \\
\hline Le 14 novembre & 9,51 & & 26,53 & 21,37 & 21,17 & 15,29 & 15,60 & 17,72 \\
\hline Le 2 décembr & 19,86 & 21,26 & 24,22 & 11,13 & 20,57 & 24,53 & 20,13 & 23,53 \\
\hline Le 15 décembre. & 24,48 & 24,68 & 28,87 & 13,71 & 27,34 & 26,68 & 26,81 & 22,75 \\
\hline
\end{tabular}


B. Quantités d'azote des produits de décomposition pour 100 d'azote total.

\begin{tabular}{|c|c|c|c|c|c|c|c|c|c|}
\hline & \multirow[t]{2}{*}{$\begin{array}{l}\text { Date } \\
\text { déterminations }\end{array}$} & \multicolumn{2}{|c|}{ Fromage témoin } & \multicolumn{2}{|c|}{$\begin{array}{l}\text { Fromage avec } \\
1 \text { partie de pepsine } \\
\text { 1 our } 1.000 \text { parties } \\
\text { de caillé frais }\end{array}$} & \multicolumn{2}{|c|}{$\begin{array}{c}\text { Fromage aree } \\
2 \text { parties de pepsine } \\
\text { pour } 1.000 \text { parties } \\
\text { de caillé frais }\end{array}$} & \multicolumn{2}{|c|}{$\begin{array}{l}\text { Fromage avee } \\
\text { 3 parties de pepsine } \\
\text { pour } 1.000 \text { parties } \\
\text { de caillé frais }\end{array}$} \\
\hline & & Extérieur & Noyau & Extérieur & Noyau & Extérieur & Noyau & Extérieur & Noyau \\
\hline Le & 1 novembre & 1,49 & 1,12 & 2,35 & 2,29 & 1,74 & 0,93 & 3,55 & 2,47 \\
\hline Le & 14 novembre & 5,80 & 4,41 & 12,21 & 11,30 & 9,86 & 7,22 & 8,75 & 10,59 \\
\hline Le & 2 décembre.. & 7,25 & & 7,33 & 6,64 & 8,75 & 8,80 & 6,64 & 7,84 \\
\hline$L_{\theta}$ & 15 décembre. & 9,04 & 9,48 & 8,07 & 7,06 & 11,87 & 8,75 & 10,00 & 9,42 \\
\hline
\end{tabular}

\section{Quantités d'azote ammoniacal pour 100 d'azote total.}

\begin{tabular}{l||r|r|r|r||r|r||r|r} 
Le 1 novembre & 1,16 & 1,18 & 1,04 & 1,12 & 1,00 & 0,60 & 1,46 & 1,52 \\
Le 14 novembre & 2,09 & 2,30 & 2,02 & 2,58 & 3,19 & 2,97 & 2,13 & 2,44 \\
Le 2 décembre. & 3,79 & 3,84 & 2,58 & 2,77 & 2,74 & 3,00 & $-3,15$ & 3,76 \\
Le 15 décembre. & 4,11 & 4,94 & 4,23 & 3,92 & 4,91 & 4,37 & 4,24 & 4,11 \\
\hline \hline
\end{tabular}

Les résultats des tableaux VII et VIII sont rassemblés dans les tableaux suivants.

\section{TABLEAU IX}

Fromages fabriqués le 12 avril.

\begin{tabular}{|c|c|c|c|c|c|c|c|c|c|}
\hline \multirow{2}{*}{ 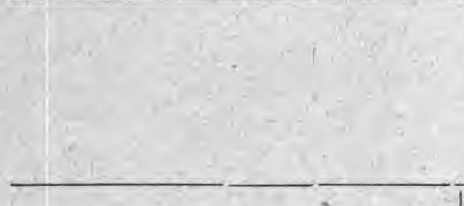 } & & & \multicolumn{2}{|c|}{ Azote soluble } & \multicolumn{2}{|c|}{$\begin{array}{c}\text { A7ote des } \\
\text { produits de } \\
\text { désagrégation }\end{array}$} & \multicolumn{2}{|c|}{$\begin{array}{l}\text { A zote am- } \\
\text { meniacal }\end{array}$} \\
\hline & & & & $\begin{array}{c}\text { Exté- } \\
\text { rieur }\end{array}$ & Noyau & $\begin{array}{l}\text { Exté- } \\
\text { rieur }\end{array}$ & Noyau & 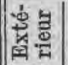 & 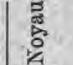 \\
\hline & Dans & 15 & jours & & & & & & \\
\hline Fromage témoin & & & 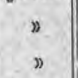 & $\begin{array}{l}44,68 \\
44,16\end{array}$ & & $\begin{array}{l}25,79 \\
25,89\end{array}$ & $\begin{array}{l}19,06 \\
20,52\end{array}$ & $\begin{array}{l}0,25 \\
6,43\end{array}$ & $\begin{array}{l}0,17 \\
5,30\end{array}$ \\
\hline $\begin{array}{l}\text { Fromage avec } 0,5 \text { partie de pep- } \\
\text { sine pour } 1.000 \text { parties de caillé } \\
\text { frais }\end{array}$ & $\left\{\begin{array}{c}\text { Dans } \\
n \\
\eta\end{array}\right.$ & $\begin{array}{l}15 \\
36 \\
49\end{array}$ & $\begin{array}{c}\text { jours } \\
n \\
n\end{array}$ & & $\begin{array}{r}4,31 \\
22,08 \\
43,24\end{array}$ & $\begin{array}{r}6,37 \\
39,40 \\
44,95\end{array}$ & $\begin{array}{r}0,59 \\
12,75 \\
22,36\end{array}$ & $\mid \begin{array}{l}0,99 \\
0,55 \\
6,09\end{array}$ & $\begin{array}{l}0 \\
0,22 \\
8,19\end{array}$ \\
\hline Fromage avec 1,0 partie de pepsine & Dans & 15 & jours & 15,25 & 0,18 & 2,06 & 1,36 & 0,05 & 0 \\
\hline pour 1.000 parties de caillé & x & 36 & $n$ & 56,11 & 18,12 & 38,35 & 13,03 & 0,18 & 0,28 \\
\hline frais & ” & 49 & $n$ & 76,18 & $3^{\prime} 66,94$ & 48,48 & 23,81 & 5,72 & 7,52 \\
\hline Fromage avec 1,5 parties de pep- & Dans & 15 & jours & 21,31 & 4,22 & 7,74 & 2,02 & 0,44 & 40 \\
\hline sine pour 1.000 parties de caillé & \# & 36 & " & 59,11 & 19,74 & 429,71 & 17,80 & 0,42 & 0,21 \\
\hline frais & $n$ & 49 & $"$ & - & - & - & - & - & - \\
\hline
\end{tabular}


TABLEAU $\mathrm{X}$

Fromages fabriqués le 23 octobre.

\begin{tabular}{|c|c|c|c|c|c|c|c|}
\hline & & Azcte : & soluble & $\mid \begin{array}{l}\text { Azote } \\
\text { produi } \\
\text { désagr } \\
\text { tio }\end{array}$ & $\begin{array}{l}\text { des } \\
\text { ts de } \\
\text { éga- } \\
\text { n }\end{array}$ & & \\
\hline & & $\begin{array}{l}\text { Exté-' } \\
\text { rieur }\end{array}$ & 'Noy & \begin{tabular}{|l} 
Exté- \\
rieur
\end{tabular} & है & 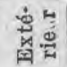 & 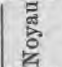 \\
\hline & & & 1 & & & & \\
\hline & Dans 15 jours & 7,13 & - & 4,31 & $|3,29|$ & $\{0,93$ & 1,12 \\
\hline Fromage témoin & Dans 1 mois & 17,48 & 20,01 & 5,76 & - & 2,63 & 2,66 \\
\hline & " $1 \frac{1}{2}$ mois & 22,10 & 23,43 & 7,55 & 8,36 & $\mid 2,95$ & 3,76 \\
\hline Fromage avec 1,0 partie de pep- & Dans 15 jours & 22,35 & 17,13 & 9,86 & 9,01 & 0,98 & 1,46 \\
\hline sine pour 1.000 parties de caillé & 1 mois & 20,04 & 6,89 & 4,98 & 4,35 & $5 \mid 1,54$ & 1,65 \\
\hline frais & " $11 / 2$ mois & 24,69 & 9,47 & 5,72 & 4,77 & $\begin{array}{l}7 \\
7,19\end{array}$ & 2,80 \\
\hline Fromage avee 2,0 parties de pep- & Dans 15 jours & 17,58 & 14,16 & 8,12 & 6,29 & 2,19 & 2,37 \\
\hline sine pour 1.000 parties de caillé & „1 mois & 16,98 & 23,40 & 7,01 & 7,87 & 71,74 & 2,40 \\
\hline frais & $1 \frac{1}{2}$ mois & s 23,75 & 525,55 & 10,13 & 7,82 & 23,91 & 3,77 \\
\hline Fromage avec 3,0 parties de pep- & Dans 15 jours & 89,51 & 13,92 & 5,20 & 8,12 & 20,67 & 0,92 \\
\hline sine pour 1.000 parties de eaillé & "1 mois & 14,04 & 19,73 & 3,09 & 5,37 & $7 \quad 1,69$ & 2,24 \\
\hline frais & " $1 \frac{1 / 2}{2}$ mois & s 20,72 & 218,95 & 6,45 & 6,95 & 52,78 & 2,59 \\
\hline
\end{tabular}

Voyons les conséquences qu'ont tirées Marcas et HuYGe.

A la fin de leur article, ils écrivent : "Cette solubil sation des matières album.noïdes n'est pas proportionnelle à la quantité de pepsine ajoutée ; elle est surtout prononcée pendant les premières semaines qui suivent la fabrication, lorsque l'acidité de la pâte est élevée ».

Plus loin, suit la conclusion qui se dégage moins des résultats de l'expérience, que des op.nions qui dominaient en ce temps-là sur le rôle de la pэpsine dans le processus de la maturation des fromages : " La con lusion qui se dógage de ces résultats, c'est que la pepsine joue un rôle très utile dans la maturation des fromages de Herve ".

Mais si la haute teneur en comb.naisons azotées solubles est une chose, une tout autre chose est la haute qualité du fromage. Et nous voilà alors, en plein acsord avec l'op.nion de L. van SLYKE et B. HART (1903) déjà məntionnée; pour ces auteurs, "c ctte conclusion est cependant en d́saccord avec les jugements des praticiens. Les appréciations des gens de la pratique, qui ont examné l'odeur, le goût, l'élasticité etc., de nos fromages, ne concordaient pas avec les résultats analytiques. ”

Par la suite, nous reviendrons encore sur le travail de Marcas et HUYGE.

E1 1979, le rhim iste hollandais van DAM [13] a publié dans le journal Hopp-Seyler's Zeitschrift für physiologische Chemie, une étude : Ueber die Wirkung des Lab's auf Parakaseinkalk ». (1) L'année suivante, ce

(1) Sur l'action du lab sur le paracaséinate de chaux. 
travail avec un supplément concernant la maturation du fromage a été reproduit presque sans changement dans le journal Centralblatt für Bakteriologie, sous le titre "Enzym-chemische Studien über die Edamerkảsereifung " (1) [14]. Dans ces deux travaux, van DAM a donné pour la première fois une représentation exacte de l'acidité véritable du fromage.

Tandis que quelques auteurs se basant sur les données obtenues par titrémétrie ordinaire, trouvaient dans le fromage de grandes quantités, environ $1 \%$ d'acide lastique, qui étaient plus que suffisantes pour l'activation de la pepsine, van DAM a montré à l'aide de l'élément concentrateur, construit par lui, que l'acidité véritable de la masso fromagère est moins que $\frac{\mathrm{N}}{100.000} \mathrm{HCl}$. En outre, il a montré qu'en l'appliquant sur du papier $\mathrm{C}$ ongo qui devient bleu à $\mathrm{C}_{\mathbf{H}}=1.10^{-4}$, il ne variait pas de couleur, tandis qu'une solution d'acide lactique, d'une richesse moindre, titrimétriquement parlant, fait virer au bleu aussitôt le papier Congo.

Ayant appris à déterminer le degré d'acidité exacte, acidité actuelle dirons-nous aujourd'hui, van DAM crut possible de fixer par des expériences directes, si le lab joue un rôle dans le processus de la maturation du fromage, et quel est ce rôle. Dans ce but il a entrepris l'étude de l'action du lab sur des préparations de paracaséine.

Il est connu que quelques physiologistes : Pekelharing, Pavloff, ont avancé l'opinion que le lab-ferment est identique à la pepsine; c'est le point de vue uniciste; d'autres expérimentateurs, (principalement l'école de 0 . Hammarsten) émettent l'opinion qu'à côté de a pspsine existe un autre ferment, la chymosine, qui agit à un degré très bas d'acidité; c'est le point de vue dualiste.

Dans ses travaux de 1909-1910, van DAM a essayé d'adopter une solution intermédiaire. Pour éclaircir le caractère de l'action du lab après la coagulation, l'auteur, avant toute chose, a fait porter ses recherohes sur la digestion du paracaséinate de calcium par le lab aux différentes concentrations en ions $\mathrm{H}$.

Pour cela, il employait la méthode suivante :

5 litres de lait écrémé étaient coagulés à $30^{\circ} \mathrm{C}$. en $15 \mathrm{~min}$. Le caillé reçu était traité aussitôt par une quantité quadruple d'eau froide. Ensuite la masse était laissée au repos, puis décantée. Cette opération était répétée six fois. Puis la masse était filtrée à travers la mousseline et la partie retenue était triturée avec de l'alcool. Après la filtration et le lavage par al cool à $96 \%$, le produit était traité par l'éther. Comme résultat, on obtenait une poudre fine qui se composait de paracaséinate du calcium et des phosphates inso ubles du lait.

(') Etudes enzymo-chimiques sur la maturation du fromage d'Edam. 
Dans 8 eapsules, on plaçait 4 gr. de paracaséinate pour chacune. Puis on y versait, $35 \mathrm{~cm}^{3}$ de $\mathrm{HCl}$ dilué à diverses concentrations et on ajoutait $80 \mathrm{mgr}$. de thymol. Les capsules étaient placées 24 heures dans l'étuve à $25^{\circ}$ et agitées lentement; puis on versait dans chacune $5 \mathrm{~cm}^{3}$ d'une solution de lab; ces capsules étaient agitées de nouveau pendant 42 heures. Dans quatre autres capsules témoins, on ajoutait aussi $5 \mathrm{~cm}^{3}$ de lab, mais ce dernier était préalablement tué par l'ébullition pendant 3 heures. Après 66 heures, toutes les dissolutions étaient filtrées et dans le filtrat on déterminait l'azote d'après KJELDAHL et on mesurait la concentration en ions $\mathrm{H}$.

Les résultats des expériences sont présentés dans le tableau XI.

TABLEAU XI

\begin{tabular}{|c|c|c|c|c|}
\hline & $\mathrm{C}_{\mathrm{H}} \times 10^{-6}$ & $\begin{array}{l}N_{1}\left(\mathrm{~cm}^{3} \text { d'acide }\right. \\
1 / 10 \text { normal) }\end{array}$ & $\begin{array}{l}N \text { dans la capsule } \\
\text { témoin }\end{array}$ & Digéré \\
\hline 1. & 0,105 & 12,2 & 1,7 & 10,5 \\
\hline 2. & 0,33 & 20,2 & 2,4 & 17,8 \\
\hline 3. & 0,78 & 27,6 & 3,2 & 24,4 \\
\hline 4. & 1,36 & 28,9 & 2,6 & 26,3 \\
\hline
\end{tabular}

On voit que le paracaséinate de calcium se dissout par le lab, et que l'action de l'enzyme croît fort avec la concentration croissante en ions hydrogène. Pourtant, il arrive un moment ou la concentration des ions $\mathrm{H}$. est telle que les produits de la décomposition empêchent l'action du ferment. C'est pourquoi si l'on traduisait les résultats graphiquement, en mettant en abscisse, les grandeurs des concentrations des ions $\mathrm{H}$ et en ordonnée les quantités de protéine digérée, la courbe tendrait de plus en plus à aller parallèlement à l'axe des X.

Après avoir fixé l'action dissolvante du lab sur le paracaséinate de calcium, van DAM s'est donné pour but d'éclaircir la relation entre le pouvoir coagulant $\mathrm{du}$ lab sur le lait et son pouvoir dissolvant de la paracaséne. L'auteur raisonnait ainsi : si la pepsine est la cause de la digestion, il n'est pas vraisemblable que les capacités coagulantes et digérante soient parallèles chez les différentes préparations du lab.

Citons un extrait du résumé de l'article, où l'auteur, formulant les résultats de ses expériences déelare :

" En donnant aux différentes préparations de lab une acidité égale pour agir sur le paracaséinate du calcium, les vitesses de digestion sont tout à, fait parallèles aux vitesses de coagulation du lait ». Le même résultat a été trouvé pour les solutions de lab préparées d'après HaMMARSTEN et les macérations d'estomac du veau danslesquelles la pepsine a été affaiblie par un traitement par le carbonate de magnésium.

De là, on peut tirer la conclusion qu'avec des concentrations en 
ions H jusqu'à $10-^{5}$ comme e'est le cas dans mes dissolutions, il peut à peine s'agir de l'action de la pepsine; la paracaséine ne se dissoudrait que sous l'action de la chymosine. Il n'y a aucune raison d'admettre, selon Petry, l'existence dans le lab d'une enzyme inconnue spécifique pour la caséine.

Faisons une description d'une des expériences qui illustrent le parallélisme de la vitesse de la coagulation du lait et celle de la digestion du parazaséinate de calcium.

On dissout 20 gr. de lab en poudre de HANSEN dans $180 \mathrm{~cm}^{3}$ d'eau et on les dialyse contre l'eau de pluie. On agite pendant 5 minutes $120 \mathrm{~cm}^{3}$ de la dissolution assez claire, avec $1 \mathrm{gr} .2 \mathrm{de} \mathrm{MgCO}^{3}$ et on filtre rapidement; on répète cette opération deux fois. Le liquide obtenu est ainsi neutralisé.

La dilution de $1: 4$ de la solution initiale en présence de $\mathrm{HCl}$ à $0,1 \%$ digère un brin de fibrine en 2 heures, tandis que le liquide neutre obtenu comme il vient d'être dit exigeait 18 heures pour dissoudre à peine les $2 / 3$ du brin. D'autre part, la relation des vitesses de coagulation était $35 \mathrm{sec}$., $207 \mathrm{sec}$. Le tableau suivant contientles résultats de l'action de ces dissolutions sur le paracaséinate de calcium ; par ailleurs, le contenu du Mg dans toutes les capsules a été rendu le même.

TABLEAU XII

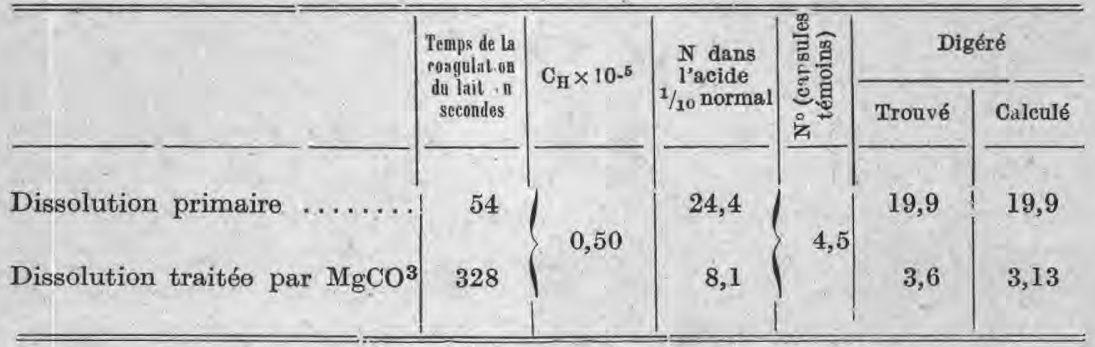

Puis l'auteur a passé à des expériences sur l'affaiblissement de la chymosine d'après Hammarsten. Ce n'est que de cette manière qu'on paut se rendre compte si la pepsine prend part et dans quelle mesure à la dissolution de la paracaséine aux concentrations faibles en ions $\mathrm{H}$.

Les résultats de ces expériences sont exposés dans le résumé de l'article, où l'auteur s'exprime ainsi :

" En détruisant dans la macération de l'estomac du veau la chymosine par digestion avee $\mathrm{HCl}$ à $0,2 \%$, la capacité de digérer la masse fromagère disparaît presque tout à fait, même avec une acidité de $1,4 \cdot 10_{-}^{5}$. Dans $\mathrm{HCl}$ à $0,2 \%$, une solution pareille digère visiblement le blanc d'œuf de la poule. Au moyen des expériences spéciales avec l'enzyme de l'estomac de cochon purifiée d'après Pekrlharing on a constaté qu'on doit attribuer à la pepsine la digestion insignifante de la matière 
fromagère par la macération de l'estomac du veau, additionné d'acide chlorhydrique. Dans le lab ordinaire, l'action de la pepsine est tellement en arrière de celle de la chymosine, qu'on observe toujours un plein parallélisme entre la vitesse de la coagulation du lait et celle de la digestion de la matière fromagère 1.

Faisons une description de l'expérience mentionnée avec l'enzyme purifiée d'après Pekelharing.

On a préparé une macération de l'estomac du veau qu'on faisait digérer avec $\mathrm{HCl}$ à $0,2 \%$ jusqu'à ce que le rapport des vitesses de la coagulation du lait fût de $80 \mathrm{sec}$., 3 heures $45 \mathrm{sec}$. La capacité coagulante a été mesurée d'après la méthode Metr. Puis, on fit une dissolution de l'enzyme de l'estomac du cochon dans de l'HCl à $0,2 \%$, et on la dilua jusqu'à ce que les grandeurs de la digestion d'après METT, fussent égales pour les enzymes du cochon et du veau ; c'est alors qu'on procéda à l'épreuve de la digestibilité de la paracaséine.

TABLEAU XIII

\begin{tabular}{|c|c|c|c|c|}
\hline & $\mathrm{O}_{\mathrm{H}} \times 10-5$ & $\begin{array}{l}\mathrm{N}_{1 / 10}\left(\mathrm{~cm}^{3} \mathrm{~d}^{\mathrm{r}} \text {, cidormal }\right) \\
\text { norme }\end{array}$ & $\begin{array}{l}\text { N (capsules } \\
\text { témoins) }\end{array}$ & Digéré \\
\hline Enzyme du cochon..... & 0,72 & 14,1 & 7,4 & 6,7 \\
\hline Enzyme du veau ...... & & 16,6 & 9,4 & 7,2 \\
\hline
\end{tabular}

Ainsi, deux solutions qui agissent avec une force égale sur le blane d'ouf de poule, dissolvent également la caséine. Par conséquent, le parallélisme trouvé plus haut de la coagulation du lait et de la digestion du paracáséinate est la suite de ce que la digestion par la pepsine est un plan en arrière de celui de la digestion par la ehymosine.

Pour appliquer les données obtenues sur l'action du lab sur le paracaséinate pur de calcium au processus de la protéolyse de la masse fromagère, il faut tout d'abord montrer que le lab-ferment se trouve dans le fromage dans un état actif. En effet, van DAM a réussi à constater la présence de la chymosine adsorbée dans le fromage à l'âge de 3 semaines.

Pour cela $200 \mathrm{gr}$. de fromage étaient triturés avec de l'eau, le volume était porté jusqu'à $1 / 2$ litre et le liquide obtenu était maintenu environ 4 heures à $25^{\circ} \mathrm{C}$. Après filtration, $100 \mathrm{~cm}^{3}$ de la dissolution étaient évaporés dans le vide à $35^{\circ}$ jusqu'à l'état de gruau. Cette masse était mélangée avec $20 \mathrm{~cm}^{3}$ d'eau et dialysée pendant 24 heures contre $\mathrm{HCl}$ à $0,2 \%$. L'albumine se séparait sous une forme facile à filtrer et l'enzyme restait dissoute. Le liquide opalescent après la filtration était de nouveau dialysé contre l'eau de pluie jusqu'à réaction faiblement acide, le liquide devenait trouble. Pour dissoudre le ferment, on ajoutait 
un peu de $\mathrm{NaCl}$ et on évaporait la dissolution obtenue dans le vide à $35^{\circ} \mathrm{C}$. jusqu'au volume de $5 \mathrm{~cm}^{3} ; 10 \mathrm{~cm}^{3}$ de lait avec deux gouttes de chloroforme étaient coagulés par $1,5 \mathrm{~cm}^{3}$ de cette dissolution en une nuit à $25^{\circ} \mathrm{C}$. Le degré de l'acidité titrable était 18. A ce taux il ne pouvait s'agir d'une coagulation acide. En outre, on avait pris un autre échantillon avec $1,5 \mathrm{~cm}^{3}$ de la dissolution bouillie; cet échantillon possédait aussi une acidité de 18, mais le lait resta tout à fait liquide.

Ainsi, van DAM est arrivé à cette conviction que l'enzyme coagulante continue son action, c'est-à-dire dissout la matière du fromage. Les produits ainsi obtenus forment un milieu nutritif nécessaire pour les microorganismes, qui élaborent d'ailleurs les substances aromatiques grâce auxquelles le fromage possède une odeur et une saveur typiques.

Pour contrôler cette thèse, l'auteur a fait des expériences avec des fromages faits de lait aseptique. Ces fromages murissaient extrêmement lentement. Ainsi, l'un d'eux contenait la même quantité des combinaisons azotées solubles à l'âge de $1 \frac{1}{2}, 4$ et 8 mois. Il en tire la conclusion suivante :

« Dans les fromages préparés avec du lait aseptique, la paracaséine se dissout avant d'atteindre l'état d'équilibre. Cet état d'équilibre est semblable à celui qui est atteint quand le paracaséinate de calcium est agité avec la solution de lab. Ce n'est qu'après la dislocation de cet équilibre qu'a lieu l'augmentation de la quantité d'azote soluble dans le fromage. Le changement d'équilibre dépend probablement des microorganismes ou des enzymes formées par eux, lesquelles font subir une simplification ultérieure aux produits de décomposition qui s'étaient formés. Alors le lab-ferment peut de nouveau dissoudre la matière fromagère. "

Bien que dans l'article de van DAM, l'opinion ne soit pas exprimée nettement sur l'identité de la pepsine et de la chymosine, et que, en outre, l'auteur, sans fondements justes, ait limité le rôle des microorganismes acido-lactiques, escomptant qu'ils sont incapables de peptoniser la paracaséine, cet article a sans nul doute apportédes aperçus nouveaux sur les processus physico-chimiques, qui accompagnent la protéolyse de la masse du fromage.

En 1912 a paru un travail des collaborateurs de la Station agricole expérimentale du Wisconsin : E. G. Hastings, Alice C. Evans et E. B. HART [17], qui négligent tout à fait les faits fixés par van DAM. Dans la partie concernant l'action du lab, le travail des expérimentateurs de Wisconsin ne s'élève pas au-dessus du niveau des expériences de 1900. Voulant expliquer la peptonisation de la masse fromagère par l'action de la pepsine, activée par l'acide lactique secrété par les microbes, les auteurs ont fait l'expérience suivante :

Un lait très pur, n'ayant après 24 heures de conservation à $20^{\circ} \mathrm{C}$. 
qu'une acidité qui valait $21^{\circ} \mathrm{T}$. voit cette acidité portée au moyen d'acide chlorhydrique à $27^{\circ} 8 \mathrm{~T}$. On le fait coaguler et on en prépare un fromage. La décomposition du lactose dans ce fromage s'effectue extrêmement lentement. Tandis que dans le fromage normal, le lactose disparaît au bout de 3 à 5 jours, dans le fromage ei-dessus, il est resté après 22 jours plus de la moitié du lactose, qu'il contenait à l'âge d'un jour. $\mathrm{Au}$ bout de trois mois, le fromage n'était pas mûr et possédait une très faible odeur de fromage.

Nous voyons que cette expérience ne semble pas appuyer l'opinion de Van DaM. Il est difficile, en effet, de ne pas convenir qu'elle est loin de confirmer l'opinion des auteurs précités sur le faible rôle des microorganismes qui forment l'acide lactique. Evidemment, la cause de la faible protéolyse est moins dans la petite quantité d'acide lactique présent que dans le faible développement de la micrcflore, laquelle n'a pas été en état même en 22 jours de convertir la moitié du lactose en acide lactique; pour cette raison, la dislocation de l'équilibre dont parle van Dam n'a pu avoir lieu bien que l'enzyme du lab fût présente.

Deux ans après, Alice C. Evans et E, G. Gastings [20] au VI Congrès International de Laiterie à Berne ont avoué que l'activité des bactéries acido-lactiques "ne dépend point de la présence du lactose, ce qu'on admettait jusqu'alors ", puisque ce groupe des microorganismes peut se développer intensément dạns un milieu ne contenant que des matières propres au fromage mûr, dans lequel le lactose a disparu il y a déjà longtemps.

En 1912, van DaM [18] a publié un nouveau travail " Die Verdauung des Kaseins durch Pepsin vom Kalb, Schwein und Rind " (1) consacré à la question de l'action des enzymes de l'estomac sur la caséine.

Dans ce travail, l'auteur a admis définitivement la théorie de l'unicité de la pepsine et de la chymosine, s'appuyant sur ce que les conditions de l'expérience avec l'épreuve de METT ne sont pas comparables avee celles de l'épreuve de la coagulation du lait.

Ainsi, avec l'épreuve de METT, on travaille avec une assez grande quantité d'enzyme et à une assez haute acidité ; pour la coagulation du lait, on n'emploie que de petites quantités d'enzyme par rapport à la quantité du substratum et avec une réaction neutre ou très faiblement acide.

Ayant trouvé qu'avec un degré faible d'acidité - auquel la caséne ne se dissout pas - la vitesse de la coagulation du läit par les enzymes de l'estomac est parallèle à leur capacité de digérer la caséine, l'auteur n'a pas découvert ce parallélisme entre la vitesse de la ccagulation du

(I) La digestion de la caséine par la pepsine du veau, du cochon et du bétail. 
lait et celle de la digestion de la caséine avec $\mathrm{HCl}$ à $0,3 \mathrm{n}$ en ayant recours à l'épreuve de METt.

L'auteur a expliqué ce phénomène,non par ce que dans le cas présent e'est une autre enzyme qui agit, mais parce que, en admettant l'identité des enzymes, le système, dans lequel ils agissent, n'est pas identique (les mots ont été soulignés par l'auteur).

Citons une des expériences de van DaM, expliquée par Iui, au bénéfi e de l'unicité. On y compare l'action digérante de la macération de l'estomac du veau et celle de la dissolution de la pepsine dans $\mathrm{HCl}$ normal à $0,3 \% \mathbf{n}$ ainsi qu'en présence d'acétate de scdium en vue de l'abaissement de la concentration en ions $\mathrm{H}$.

TABLEAU XIV

\begin{tabular}{|c|c|c|c|c|c|c|c|c|}
\hline & & $\underbrace{\mathrm{Hel}}_{3^{32} / 10}$ & acide & & $\underbrace{}_{\mathrm{m}^{31 / 10}} \mathrm{n}$. & $\frac{\text { odium }}{\text { acide }}$ & $\begin{array}{c}\text { Temps } \\
\text { de I } \\
\text { coagula- }\end{array}$ & $\begin{array}{l}\text { Digéré } \\
\text { d'après Mett. }\end{array}$ \\
\hline & & $\begin{array}{l}\text { Bupér nee } \\
\text { temoin }\end{array}$ & Digéré & & $\begin{array}{l}\text { bxyérienre } \\
\text { lemain }\end{array}$ & Digéré & & \\
\hline Enzyme du veau & 10,4 & 3,2 & 7,2 & 24,5 & 2,2 & 22,3 & $81^{\prime \prime}$ & $3,0 \mathrm{~mm}$. \\
\hline Enzyme du cochon & 38,2 & 3,2 & 35,0 & 27,8 & 2,2 & 25,6 & $74^{\prime \prime}$ & 5,8 $\mathrm{mm}$ \\
\hline
\end{tabular}

On voit que l'enzyme du veau ainsi que celui du cochon digéraient bien d'après l'épreuve de METT et coagulaient le lait; l'enzyme du cochon même un peu mieux, puisqu'elle agissait même plus intensément que l'enzyme du veau en milieu faib_ement acide.

Pourtant, à examiner avec attention le tableau XIV, il n'est pas difficile de conclure autrement que l'auteur. Ainsi l'enzyme du veau digérait trois fois moins de caséine en milieu faiblement acide que l'enzyme du cochon. Evidemment, il est plus logique d'avancer que dans la macération de l'estomac du veau domine la chymosine, ce ferment protéolytique qui agit préférablement en milieu faiblement acide, tandis que dans les préparations obtenues des estomacs du cochon domine une autre enzyme : la pepsine qui développe son action principalement en milieu plus acide.

En 1915, van Dam [21] a publié un nouvel article sous le titre "Die Pepsin-Chymosin-Frage und die Käsereifung " (1).

Duns ce travail qui est plutôt une revue, consacrée à la preuve de l'identité des enzymes de l'estomac, se trouve une remarque dans laquelle l'auteur confirme que dans sa fromagerie expérimentale on avait obtenu d'excellents fromages d'Edam en n'employant pour leur fabrication que (souligné par l'auteur) de la pepsine, c'est-à-dire une

(1) La question de la pepsine et de la chymosine et la maturation des fromages. 
solution qui ne coagulait pas le lait non acidulé même au bout de $3 \frac{1}{2}$ heures à la temṕrature la plus favorable pour la coagulation.

Q xoique l'auteur au bout de son article ait déclaré : " Es war aber keineswegs meine Absicht eine erschöpfende Uebersicht über diese interessante Frage zu geben » (1), une expérien si importante exigeait plus de développəment; aussi l'absence complète des indications teshniques nézessaires en dépréc e la valeur. C'est ainsi que la quantité de $p э p$ sine utilisée n'est pas indiquée; on ne dit pas de quelle manière a été obtenue le caillé. Si c'est par acidulation forte du lait, alors cela change tout à fait les conditions physico-chimiques de la maturation du fromage. Il reste à supposer que l'auteur se servait d'une culture très active de microorganismes lactiques, qui se sont montrés capables de pəptoniser à un degré suffisant les albumines du fromage malgré la quantité évidemment insuffisante de lab.

Le rôle des microorganismes lactiques dans le processus de la maturation du fromage se présentait à diverses reprises comme thème des expériences. Mais ce n'est que récemment qu'on a réussi à montrer que les bactéries lactiques, qui appartiennent au groupe Streptococcus lactis, possèdent une capacité assez considérable de désagréger la caséine. Ce mérite appartient au bactériologiste suédois Chr. BARTHEL [22] qui a publié en 1915 un article sous le titre : "Das kaseinspaltende Vermözen von zur Gruppe Streptococcus lactis gehörenden Milchsäurebakterien » (2).

(A suivre.)

\title{
QUELQUES NOTES DE LAITERIE SUR L'ALGÉRIE
}

\author{
par C. BAN DANOU.
}

Docteur Vétérinaire, Ancien Répátıteur de Zootıchnie à l'Ecole Nationale d'Agric Itture de Montpellier; Service de l'Elevage dy l'Algérie à Miliana.

Dans les steppes du sud algérien où $n$ madisent les 9/10 du cheptel ovin, le lait de brebis sert en partie à l'élevage des jeunes, mais surtout à l'alimentation des tribus pastorales. Celles-ci en extraient d'abord le beurre (,) et consomment le petit-lait après fermentation lactique, soit en nature, soit sous forme de caséine desséchée ou klila.

Lors des années pluvieuses, lorsque l'herbe ne manque pas dans les steppes, les agnelages s'échelonnent sur les quatre saisons :

$\begin{array}{lll}R ' b i i & \text { agnelages de printemps } \\ \text { Saï i } & \text { " } & \text { d'été } \\ \text { Kherfi } & \text { " } & \text { d'automne } \\ \text { Chetoui } & \text { " } & \text { d'hiver }\end{array}$

(1) Mais je n'ai, en aucune manière, l'intention de donner un aperçu définitif sur cette question intéressante.

(2) La capacité de désagréger la caséine des bactéries acido-lactiques du groupe Strep. tococcus lactis.

(3) "Industrie beurrière ehez les nomades des Hauts-Plateaux ", par BeN Danou (Bulletin de l'Office Colonial de l'Algérie, année 1911). 